\title{
Shantala e os benefícios para desenvolvimento físico, emocional e comportamental dos bebês
}

\author{
Shantala and its Benefits for Babies' Physical, Emotional and Behavioral Development
}

\author{
Shantala y sus beneficios para el desarrollo físico, emocional y conductal de los bebés \\ Rebecea Christine Vieira Canaan ${ }^{1 *}$, Eliara Adelino da Silva ${ }^{2}$, Geísa Sereno Velloso da Silva ${ }^{3}$, \\ Alessandra da Silva Souza ${ }^{4}$, Jannaína Sther Leite Godinho Silva ${ }^{5}$, Manoela Alves ${ }^{6}$
}

Como citar esse artigo. Canaan, RCV; da Silva, EA; da Silva, GSV; Souza, AS; SIlva, JSLG; Alves, M. Shantala e os benefícios para desenvolvimento físico, emocional e comportamental dos bebês. Revista Pró-UniverSUS. 2021 Jul./Dez.; 12 (2)SUPLEMENTO: 53 - 57

\section{Resumo}

Objetivo: Este estudo tem como objetivo identificar os benefícios do método de Shantala e correlacionar a importância da evolução do vínculo entre mãe-bebê e o seu desenvolvimento através da massagem. Metodologia: O presente estudo trata-se de uma revisão integrativa nas bases de dados do Lilacs, Medline, Sciello, Bireme, fazendo uma busca ativa de artigos entre os anos de 2012 a 2020. Resultado: Os resultados mostram que, a Shantala proporciona uma qualidade de vida para os bebês e crianças estendendo para os pais e profissionais, pelo fato de contribuir na redução de estresse, cólicas abdominais, ajuda no fortalecimento do vínculo afetivo, do sistema imunológico, no desenvolvimento em crianças portadoras de doenças crônicas e traz equilíbrio físico e emocional. Conclusão: Neste estudo, as evidências obtidas mostram que a técnica da massagem Shantala beneficia o desenvolvimento físico, emocional e comportamental que acaba influenciando na qualidade de vida. Além disso, acaba proporcionando momentos de afeto, amor, carinho entre pais e filhos. Possibilitando as crianças, adolescentes e futuros adultos mais equilibrados, harmonizados e felizes com o mundo e consigo mesmo.

Palavras-chave: Bebês; Benefícios; Enfermagem; Massagem; Shantala.

\begin{abstract}
Objective: This study aims to identify the benefits of the Shantala method and correlate the importance of the evolution of the bond between mother and baby and its development through massage. Methodology: This study is an integrative review in the databases of Lilacs, Medline, Sciello, Bireme, through an active search for articles between the years from 2012 to 2020. Results: The results show that Shantala provides a quality of life for babies and children extending to parents and professionals, because it contributes to the reduction of stress, abdominal cramps; helps in strengthening the affective bond, the immune system, the development in children with chronic diseases and brings physical and emotional balance. Conclusion: In this study, the evidence obtained shows that the Shantala massage technique benefits physical, emotional and behavioral development that ends up influencing quality of life. Enabling children, teenagers and future adults to be more stable, reconciling and happy with the world and with themselves.
\end{abstract}

Keywords: Babies; Benefits; Massage; Nursing; Shantala.

\section{Resumen}

Objetivo: Este estudio tiene como objetivo identificar los beneficios del método Shantala y la importancia del vínculo madrebebé para el desarrollo del bebé y correar la evolución del vínculo entre madre e hijo a través del masaje Shantala. Metodología: Este estudio es una revisión integradora en las bases de datos de Lilacs, Medline, Sciello, Bireme, realizando una búsqueda activa de los años desde 2012 a 2020. Recolección: Los resultados muestran que Shantala proporciona una calidad de vida para bebés y niños que se extiende a los padres y profesionales, ya que contribuye a la reducción del estrés, calambres abdominales, ayuda a fortalecer el vínculo afectivo, el sistema inmunológico, en el desarrollo en niños con enfermedades crónicas y aporta equilibrio físico y emocional. Conclusión: En este estudio, la evidencia obtenida muestra que la técnica de masaje Shantala beneficia el desarrollo físico, emocional y conductual que termina influyendo en la calidad de vida. Además, termina proporcionando momentos de afecto y amor entre padres e hijos. Permitir a los niños, adolescentes y futuros adultos más equilibrados, armonizados y felices con el mundo y consigo mismos.

Palabras clave: Bebés; Beneficios; Enfermería; Masaje; Shantala.

\footnotetext{
Afiliação dos autores:
}

${ }^{1}$ Acadêmico do Curso de Enfermagem, Universidade de Vassouras. RJ, Brasil. E-mail: rebeccacanaan@hotmail.com ORCID: https://orcid.org/0000-0001-7415-8431

${ }^{2}$ Mestre. Professor do Curso de Enfermagem, Universidade de Vassouras. RJ, Brasil. Email: eliaraadelinos@uol.com.br ORCID: https://orcid.org/0000-0003-4387-6662

${ }^{3}$ Mestre. Professor do Curso de Enfermagem, Universidade de Vassouras. RJ, Brasil. Email: geisa.velloso@hotmail.com ORCID: https://orcid.org/0000-0003-0304-8010

${ }^{4}$ Mestre. Professor do Curso de Enfermagem, Universidade de Vassouras. RJ, Brasil. Email: alesouza22@yahoo.com.br ORCID: https://orcid.org/0000-0002-9009-9774

${ }_{5}^{5}$ Mestre. Docente do Curso de Enfermagem, Universidade de Vassouras. RJ, Brasil. Email: jjasther@gmail.com ORCID: https://orcid.org/0000-0002-8308-2093

${ }^{6}$ Docente na Universidade de Vassouras. Vassouras - RJ, Brasil. Email: alves.manoela@gmail.com ORCID: https://orcid.org/0000-0003-4239-9577

* Email de correspondencia: rebeccacanaan@hotmail.com 


\section{Introdução}

Nos dias atuais, está diminuindo o vínculo entre mãe e filho principalmente no trabalho de parto, pois as mulheres de hoje estão optando a fazer mais a cesárea que acaba mudando a fisiologia natural do parto. A Shantala acaba proporcionando um vínculo muito grande entre mãe e filho despertando através do toque de amor, carinho e ternura.

A descoberta da Shantala foi realizada pelo médico ginecologista e obstetra Frederick Leboyer que ao realizar uma viagem para a Índia se deparou com uma jovem massageando seu bebê e Frederick percebeu a cena de amor e carinho entre mãe e bebê e pediu a essa mãe para fotografá-la, capturando assim toques precisos de cada movimento.

Em meados dos anos 70 Leboyer, publicou um livro mostrando a técnica com o título Shantala para homenagear a mãe que havia se deixado fotografar nas ruas de Calcutá.

A Massagem de Shantala remete a vários benefícios entre eles a melhora do desenvolvimento motor, melhora a coordenação, aumenta o vínculo mãe-pai e bebê, promove interação entre família, traz equilíbrio físico e emocional alivia cólicas, fortalece o sistema imunológico, acarreta benefícios para o sistema respiratório, digestivo, imunológico, músculo esquelético aumenta auto estima, tranquiliza o sono estimula a maturação do sistema nervoso, por meio da função tátil e o desenvolvimento do sistema sensitivo ${ }^{1}$.

A Shantala é um processo fácil de aprender, usase somente as mãos e não necessita de instrumentos especiais além de ser de baixo custo e acarreta sensação de bem-estar e relaxamento na criança, sendo inúmeros os benefícios desta massagem.

É necessário para realização da massagem um ambiente aquecido, pois a mesma estará despida e não poderá sentir frio, certificar-se de que a criança não esteja de estômago cheio, e nem com fome. É recomendado retirar joias, anéis, pulseiras e relógios.

A pessoa que for executar a massagem deverá estar calma e relaxada, para ouvir, sentir e observar o bebê, poderá ter uma música ambiente, pode cantar ou falar com o bebê ou ficar em silêncio concentrada nos movimentos das Shantala e na interação com o seu bebê. Ao passar o óleo é necessário friccionar uma mão na outra para aquecer as mãos antes de tocar o corpo da criança.

À luz dessas considerações, delimitou-se como objeto de estudo: Este estudo tem como objetivo identificar os benefícios do método de Shantala e correlacionar a importância da evolução do vínculo entre mãe-bebê e o seu desenvolvimento através da massagem. Descrever os benefícios da aplicação do método da Shantala no desenvolvimento físico, emocional e comportamental do bebê?

Justifica-se pelos inúmeros benefícios que a Shantala oferece no contexto físicos, emocionais e comportamentais da saúde do bebê, além do estreitamento da relação no binômio mãe e bebê.

A partir dos benefícios da Shantala, surgiu o interesse de levar este conhecimento como uma estratégia de assistência de enfermagem visando o binômio mãe, pai e bebê como uma ferramenta do cuidado holístico, incentivando a novos estudos e aplicabilidades na atenção primária.

\section{Metodologia}

O presente estudo trata-se de uma pesquisa integrativa que pretende identificar os benefícios da Shantala para desenvolvimento físico, emocional e comportamental dos bebês correlacionando com o vínculo mãe e bebê.

A revisão integrativa consiste no estudo sistemático e ordenado de um determinado assunto ou tema a partir do que se encontra produzido e veiculado em materiais científicos já publicados.

Essa modalidade de investigação apresenta a finalidade de contribuir para o aprofundamento do conhecimento do tema investigado. Para essa revisão, fez-se o levantamento do material a ser analisado através de busca em bases de dados como, Scientific Eletronic Library Online (SCIELO), BDENF (Base de Dados em Enfermagem) Literatura Latino-Americana e do Caribe em Ciências da Saúde (LILACS) MEDLINE (Literatura Internacional em Ciências da Saúde).

A escolha por essas bases de dados justifica-se pela possibilidade de acesso às publicações eletrônicas na íntegra, facilitando a análise do material delimitado para este estudo.

Como estratégia para elaboração do projeto, o primeiro passo foi o planejamento através da pesquisa sistemática, leitura dos temas a serem trabalhados, e elaboração de fichamento, nome dos autores, título, edição, ano, formulação dos objetivos, estabelecimentos dos critérios para a seleção dos artigos, e análise dos artigos.

A pesquisa integrativa é desenvolvida com base em material já elaborado, constituído principalmente de artigos científicos. A coleta de dados se deu no período de setembro a dezembro de 2020 . O presente estudo será qualitativo com finalidade de ser um estudo descritiva buscando observar, registrar e analisar os benefícios indicados no uso da massagem Shantala e o binômio mãe/bebe.

A metodologia trata-se de uma revisão integrativa nas bases de dados do Lilacs, Sciello, Bireme, Medline. Entre os anos de 2012 à 2020 foram encontrados 10 artigos, após a leitura dos artigos percebeu-se a 
necessidade de ampliar os conhecimentos frente a Importância da Shantala no Desenvolvimento dos Bebês. Desses 10 artigos dos anos de 2012 e 2014 foram acrescentados pela ausência de conteúdo.

Este trabalho irá apresentar levantamento de dados e trabalho científico, fazendo uma busca ativa de artigos entre os anos de 2012 a 2020. Após a análise dos artigos foi elaborada uma revisão deste trabalho com os seguintes bases pesquisa as bases de dados Literatura Latino-Americana e do Caribe em Ciências da Saúde (LILACS), Scientific Eletrônic Library Online (SCIELO) e National Library of Medicine (PUBMED), Bireme.

\section{Resultados e Discussão}

Nos resultados foram encontrados 10 artigos, somando um percentual $1 \%$ dos quais todos estavam disponíveis na íntegra, após análise de exclusão e inclusão, foram selecionadas 6 publicações $60 \%$, sendo apresentado um panorama geral das publicações, conforme caracterização explicitada no Quadro 1.

Quadro 1. Apresentação do Título e Autor dos artigos consultados.

\begin{tabular}{|l|l|}
\hline \multicolumn{1}{|c|}{ Título } & \multicolumn{1}{|c|}{ Autor } \\
\hline Impacto da qualidade de vida de monitoras de & Faria KC; Silva TS; Figueredo GLA; \\
bebês institucionalizados antes e após a & Castro GG \\
aplicação da massagem Shantala & \\
\hline O benefício da shantala em crianças & Vieira HN \\
portadoras de síndrome de down & \\
\hline Shantala para promoção da saúde e conforto & Ribeiro-Lima TV; Cavalcante LIC \\
de bebês: revisão de literatura & \\
\hline O poder da massagem infantil: Shantala & Junqueira MSDA; Arruda RP; Ribeiro \\
\hline Relato de experiência: Shantala - massagem & Motter AA; Feblisbino I; Krupniski E; \\
para bebês - centro de educação infantil rosa & Micos AP; Veiga TP \\
\hline maria & MCP \\
\hline Massagem Shantala uma revisão integrativa & Nardo LRO; Silva SS; Marin MJS \\
\hline
\end{tabular}

Fonte: Autoria própria, 2021

Percebe-se na tabela acima, as evidências obtidas mostram que a técnica da massagem Shantala proporciona saúde e qualidade de vida não só para os bebês, mas também para quem a utiliza.

De acordo com os resultados da pesquisa de Faria et al. ${ }^{2}$, analisaram a distribuição de frequência das variáveis sociodemográficas, condições físicas e hábitos das monitoras. Utilizaram também o WHOQOL-Bref, um instrumento que avalia os quatro domínios: físico, psicológico, relações sociais e meio ambiente. Através das observações as monitoras das creches apresentavam estresse pelo desgaste do excesso de trabalho e sobrecarga psíquica e física. Após os dados coletados da amostra das 23 monitoras houve uma correlação positiva antes e após da utilização da Shantala entre os domínios específicos, exceto no físico. Este estudo fundamenta-se na promoção na melhora da qualidade de vida tanto das monitoras como dos bebês após a utilização da massagem².

Para Vieira ${ }^{3}$ o benefício da Shantala em crianças portadoras de síndrome de down estimula uma interação maior entre mãe e filho, como também melhora na execução dos movimentos, um sono mais tranquilo, melhora no desenvolvimento na linguagem, do controle cefálico, da transferência manual e da preensão manual, de tronco e de apoio para marcha e na qualidade do sono. $\mathrm{O}$ autor observou resultados positivos na declaração de muitos pais na utilização da Shantala e como a criança fica calma e menos agressiva aumentando o vínculo familiar através do toque e estimulando um desenvolvimento benéfico na criança ${ }^{3}$.

Quanto a promoção da saúde e conforto de bebês, Ribeiro-Lima; Cavalcante LIC, afirmaram que o toque da massagem é de intenção terapêutico por meio de uma comunicação não verbal, onde não há diretamente um sujeito passivo e outro ativo, entretanto existe uma troca de estímulos mútuos que se retroalimentam entre ambos. Os autores analisaram resultados dos benefícios descritos a partir da aplicação de sessões de Shantala em crianças, como por exemplo, aumento do padrão motor de $36 \%$ para $60 \%$ dos itens que foram avaliados, modificação nos valores de cortisol salivar, melhora no tônus muscular dos membros superiores e melhora no manuseio e convívio da mãe com a criança ${ }^{4}$.

Junqueira, Arruda, Ribeiro ${ }^{5}$, sua pesquisa reforçou sobre o poder da massagem infantil abordando sobre as sequências dos movimentos e o efeito da Shantala nos bebês. Em consonância é uma das terapias mais eficazes para alívio de dores e prevenção de doenças. A Shantala proporciona ao indivíduo tornar-se consciente do seu corpo como um todo, das suas tensões, respiração, cargas emocionais, melhora a nutrição dos tecidos pelo aumento da circulação sanguínea e linfática e outros benefícios físicos e emocionais. Os autores trouxeram um ponto deslumbrante dando ênfase que a Shantala é corpo e alma 5 .

Motter et al. ${ }^{6}$ trouxe sua contribuição através do relato de experiência no Centro de Educação Infantil e chegou à conclusão de que a participação dos pais é de suma importância para a realização da técnica da Shantala em casa, pois a criança permanece tranquila e acostumada com o toque, sendo assim, aprimorando a comunicação, criando e fortalecendo o vínculo afetivo $^{6}$. 
Após a análise do resultado mostrado pelos autores, pode-se observar que a Shantala possibilita a melhora significativa no desenvolvimento físico, emocional e comportamental, como por exemplo, na qualidade do sono, redução das cólicas, dores abdominais, diminuição do estresse, consciência corporal, fortalecimento imunológico, facilidade para se relacionar, reforço do vínculo afetivo e emocional.

Os estudos apontam que há uma limitação inicial foi o número de artigos, até por ser um tema pouco explorado nas pesquisas de enfermagem o que o torna relevante para ser explorado e difundido nos meios acadêmicos.

Os objetivos dos estudos que mais estiveram presentes sobre a Shantala remeteram-se aos aspectos comparativos, analíticos, exploratórios, envolvendo também ações práticas para promover melhor a comunicação e interação entre pais e filhos.

Percebe-se nos objetivos o interesse em

Quadro 2. Apresentação dos Objetivos dos artigos consultados.

\begin{tabular}{|l|}
\hline Comparar a qualidade de vida das monitoras de creche antes e após a aplicação da \\
massagem shantala nos bebês institucionalizados. \\
Analisar os benefícios da massagem shantala no tratamento de crianças portadoras da \\
sindrome de down. \\
\hline Explorar recente produção acerca dos benefícios da shantala para bebês em contextos \\
domésticos e de cuidado coletivo. \\
Promover uma melhor comunicação e interação entre pais e filhos, melhorar a \\
qualidade de vida do bebê e analisar os resultados da prática. \\
\hline Relatar a experiência do projeto de extensão universitário denominado "shantala: \\
massagem para bebês". \\
\hline Analisar o conhecimento produzido referente à prática da shantala, a fim de refletir \\
sobre a implantação desta técnica na prática da assistência de enfermagem à criança.
\end{tabular}

Fonte: Autoria própria, 2021

comprovar os múltiplos efeitos e vantagens desta massagem que embora seja uma técnica de baixo custo e fácil execução percebe-se ainda uma necessidade desvendar, aprofundar e explorar sobre os benefícios desta técnica milenar, a fim de agregar futuramente ao protocolo terapêutico e científico.

Sobressaíram mais as pesquisas bibliográficas, dados descritivos com natureza qualitativa e revisão integrativa de literatura com mais de $40 \%$.

No caso da pesquisa bibliográfica, voltada para este tipo de pesquisa torna evidente que os pesquisadores tiveram a intenção de buscar, a analisar, observar um dado fenômeno, no caso aqui a compreensão sobre a Shantala com o intento de concretizar os objetivos traçados, que neste caso em particular era o de denotar tamanha relevância e importância, sobre o tema, até
Quadro 3. Metodologias Utilizadas nos artigos.

\begin{tabular}{|l|c|c|}
\hline \multicolumn{1}{|c|}{ Metodologias Utilizadas } & Quantidade & $\%$ \\
\hline $\begin{array}{l}\text { Estudo transversal, exploratório, com } \\
\text { abordagem quantitativa. }\end{array}$ & 1 & $10 \%$ \\
\hline $\begin{array}{l}\text { Pesquisa bibliográfica, dados descritivos com } \\
\text { natureza qualitativa. }\end{array}$ & 2 & $40 \%$ \\
\hline $\begin{array}{l}\text { Trata-se de uma revisão integrativa de } \\
\text { literatura. }\end{array}$ & 2 & $40 \%$ \\
\hline Trata-se de uma pesquisa de campo. & 1 & $10 \%$ \\
\hline
\end{tabular}

Fonte: Autoria própria, 2021.

por se tratar de um tema pouco discutido nos meios científicos.

A pesquisa bibliográfica, por sua vez, engrandece, o tema escolhido e torna os pesquisadores mais aptos para discorrerem acerca do assunto, sobretudo, tornando-os hábeis para a discernir, confrontar as ideias deste ou daquele autor, independentemente de qualquer que sejam os fins que se estabeleçam por meio de certa situação comunicativa, norteando assim o assunto em discussão, pois isso dará sustentabilidade ao discurso.

Quanto a revisão integrativa que também teve um número expressivo também de $40 \%$, que no caso da Shantala demonstrou o cuidado bastante criterioso dos pesquisadores ao escolher este método para desenvolver seus estudos pois a escolha deste tipo de método produz resultados de qualidade aplicáveis na prática clínica, necessitando ser realizados de acordo com um método científico que lhes confira validade e no caso da Shantala agregaria ainda mais valor.

Pois as revisões integrativas são muito criteriosas

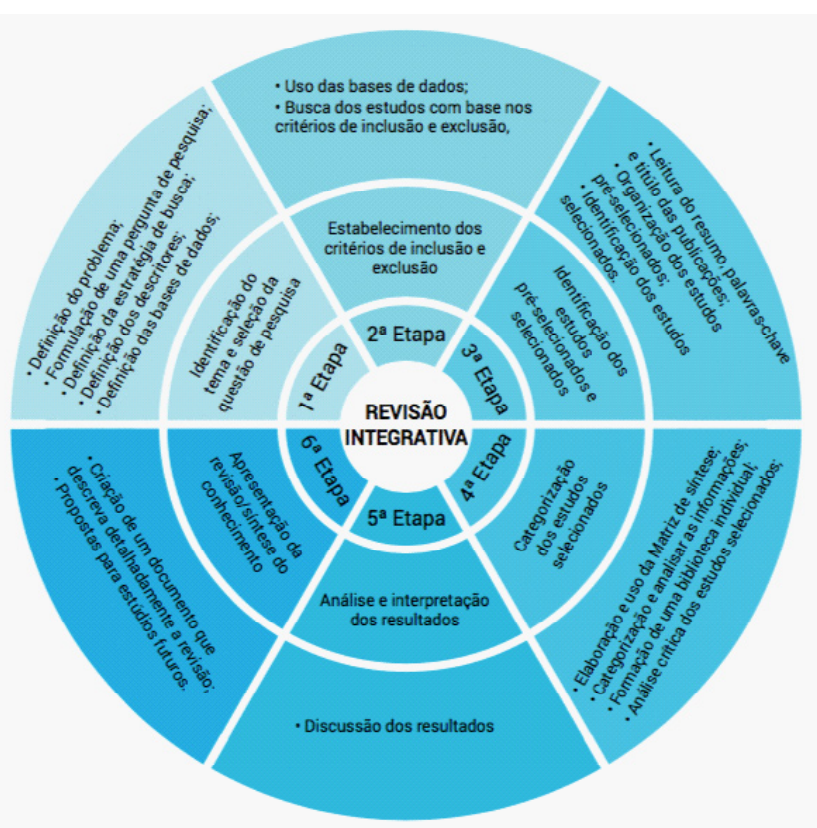

Figura 1. Processo de revisão integrativa.

Fonte: O método da revisão integrativa ${ }^{9: 129}$. 
empregadas para fornecer também os melhores conhecimentos produzidos sobre um dado tema de pesquisa, permitindo-os serem avaliados criticamente por profissional com habilidade clínica e posteriormente sejam incorporados à prática assistencial.

Este método também fornece informações mais amplas sobre um assunto/problema, constituindo, assim, um corpo de conhecimento. Deste modo, o revisor/ pesquisador pode elaborar uma revisão integrativa com diferentes finalidades, podendo ser direcionada para a definição de conceitos, revisão de teorias ou análise metodológica dos estudos incluídos de um tópico particular.

Isto explica o número expressivo desta modalidade como método de pesquisa para discutir o tema referente a Shantala.

$\mathrm{O}$ método transversal, exploratório, com abordagem quantitativa surgiu com $1 \%$ foi também utilizado pelos autores, embora em menor número sugere que na pesquisa referente a Shantala houve pesquisadores que preferiram captar as opiniões de um grupo de pessoas sobre essa técnica milenar.

Porém sugerem que essas investigações foram usadas para determinar o que acontece em tempo real, em um determinado momento como por exemplo na pesquisa intitulada Impacto da qualidade de vida das monitoras de bebês institucionalizados antes e após a aplicação da massagem Shantala podendo-se descrever as variáveis predominantes que pudessem existir em um determinado ponto e coletar assim os dados transversais.

A pesquisa de campo foi também pouco utilizada com apenas $1 \%$ que sugere poucas instituições que apresentam a prática da técnica de Shantala para ser analisada a fim de obtenção de possíveis hipóteses para a pesquisa no âmbito prático e ainda ser pouco difundido nos contextos assistenciais de saúde.

\section{Conclusão}

Neste estudo, as evidências obtidas mostram que a técnica da massagem Shantala beneficia para o desenvolvimento físico, emocional e comportamental que acaba influenciando na qualidade de vida. Além disso, acaba proporcionando um momento indiscutível de afeto, amor, carinho entre pais e filhos. Assim teremos futuras crianças, adolescentes e adultos mais equilibrados, harmonizados e felizes com o mundo e consigo mesmo.

Os resultados mostram que, a Shantala proporciona uma qualidade de vida para os bebês e crianças estendendo para os pais e profissionais, pelo fato de contribuir na redução de estresse, cólicas abdominais, ajuda no fortalecimento do vínculo afetivo, do sistema imunológico, no desenvolvimento em crianças portadoras de doenças crônicas e traz equilíbrio físico e emocional.

De maneira geral, esta pesquisa trouxe um aprendizado significativo e o desejo de compartilhar o conhecimento adquirido ao longo deste processo deslumbrante. Shantala é o corpo e alma, mostra a beleza da comunicação entre pais e filhos com troca de olhares, sorrisos e de amor. É um método alternativo de baixo custo que deve ser mais explorado e ensinado para estimular o seu uso

\section{Referências}

1. Brêtas JRS, Silva MGB. Massagem em bebês: um projeto de extensão comunitária. Acta Paul. Enf., São Paulo, 1998; 11: 59-63.

2. Faria KC, Silva TS, Figueredo GLA, Castro GG. Impacto da qualidade de vida de monitoras de bebês institucionalizados antes e após a aplicação da massagem Shantala. Revista Brasileira de Qualidade de Vida. 2018; 10 (1).

3. Vieira HN. O benefício da Shantala em crianças portadoras de Síndrome de Down. Revista Estética em Movimento. 2018; 1 (1).

4. Lima RVT. Shantala para Promoção da Saúde e Conforto de Bebês: Revisão de Literatura. Revista Eletrônica Acervo Saúde, UFPA. Belém, PA. 2020; (48)

5. Junqueira MSDA. Arruda RP. Ribeiro MCP. O Poder Da Massagem Infantil: Shantala. Revista de Iniciação Científica da Universidade Vale do Rio Verde. 2017; 6 (2).

6. Motter AA. Feblisbino I. Krupniski E. Micos AP. Veiga TP. Relato de experiência: shantala-massagem para bebês-centro de educação infantil rosa maria. Divers@!. 2012; 5(2).

7. Nardo LRO. Silva SS. Marin MJS. Massagem Shantala-Uma revisão integrativa. CIAIQ. 2014; 3.

8. Nascimento FL. Silva GSV. A falta do protagonismo da mulher no trabalho de parto-parto e nascimento. Revista Pró-UniverSUS. 2019; 10 (1): 34-37.

9. Botelho LLR, de Almeida Cunha CC, Macedo M. O método da revisão integrativa nos estudos organizacionais. Gestão e sociedade, 2011; 5(11), 121-136. 